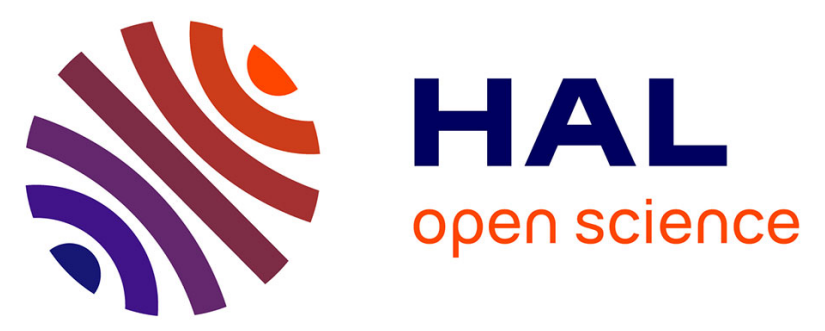

\title{
ÉTUDE NUMÉRIQUE ET EXPÉRIMENTALE DU SCHÉMA MODAL DE COQUES CYLINDRIQUES, FINIES, RAIDIES CIRCONFÉRENTIELLEMENT
}

Bernard Laulagnet, C. Boisson, M. Gotteland, B. Guerin

\section{- To cite this version:}

Bernard Laulagnet, C. Boisson, M. Gotteland, B. Guerin. ÉTUDE NUMÉRIQUE ET EXPÉRIMENTALE DU SCHÉMA MODAL DE COQUES CYLINDRIQUES, FINIES, RAIDIES CIRCONFÉRENTIELLEMENT. Journal de Physique Colloques, 1990, 51 (C2), pp.C2-253-C2-256. 10.1051/jphyscol:1990261 。 jpa-00230682

HAL Id: jpa-00230682 https://hal.science/jpa-00230682

Submitted on 1 Jan 1990

HAL is a multi-disciplinary open access archive for the deposit and dissemination of scientific research documents, whether they are published or not. The documents may come from teaching and research institutions in France or abroad, or from public or private research centers.
L'archive ouverte pluridisciplinaire HAL, est destinée au dépôt et à la diffusion de documents scientifiques de niveau recherche, publiés ou non, émanant des établissements d'enseignement et de recherche français ou étrangers, des laboratoires publics ou privés. 
COLLOQUE DE PHYSIQUE

Colloque C2, supplément au $n^{* 2}$, Tome 51, Février 1990

ler Congrès Françals d'Acoustique 1990

ETUDE NUMERIQUE ET EXPERIMENTALE DU SCHEMA MODAL DE COQUES CYLINDRIQUES, FINIES, RAIDIES CIRCONFERENTIELLEMENT ( 1 )

B. LAULAGNET, C. BOISSON, M. GOTTELAND et B. GUERIN

Laboratoire Vibrations-Acoustique, Bat. 303, INSA de Lyon, 20, Avenue Albert Einstein, F-69621 Villeurbanne Cedex, France

Résumé - Nous proposons une méthode pour déterminer le schéma modal d'une coque cylindrique finie et raidie circonférentiellement. Cette méthode débouche en particulier sur un progiciel qui permet de déterminer aisément les fréquences propres et les modes propres associés de ce type de structure.

Summary - The objective of this work is to analyse the modal scheme of a ring finite stiffened shell, and to propose a general method which require easy computations to obtain frequencies and mode shapes of this structure.

\section{I - INTRODUCTION}

L'étude des vibrations libres des coques cylindriques finies et raidies circonférentiellement à fait l'objet de nombreux travaux [1], [2], [3], [4]. Le but de notre présentation est de proposer une approche théorique de l'étude du shéma modal de ces structures qui aboutit numériquement à une démarche facile pour déterminer les fréquences propres et les nodes propres de ces structures.

\section{II - APPROCHE THEORIQUE}

L'analyse vibratoire des coques est basée sur l'approche énergétique [5]. Les hypothèses suivantes sont considérées : la coque est simplement appuyée, les raidisseurs circonférentiels peuvent avoir une section droite quelconque, et pour une coque donnée les raidisseurs peuvent ne pas avoir la mème section droite; le long de la coque les raidisseurs peuvent être régulièrement répartis ou non ; théoriquement il n'y a pas de limite pour le nombre de raidisseurs présents sur une coque. Considérons alors la fonction de Hamilton pour une coque raidie circonférentiellement par $\mathrm{N}$ raidisseurs :

$$
\psi=\int_{t_{0}}^{t_{1}} \underbrace{\left(T_{c}-W_{c}\right) d t}_{\text {coque }}+\int_{t_{0}}^{t_{1}} \underbrace{\sum_{i=1}^{N}}_{\text {raidisseurs }}\left(T_{i}-W_{i}\right) d t
$$

avec $T$ : énergie cinétique, $W$ : énergie de déformation.

a/ La fonction de Hamilton de la coque non raidie construite à partir des hypothèses de Donnell est classique. (Inertie rotationnelle et effort tranchant négligés), voir modèle sur la figure 1 .

b/ Pour ce qui concerne les raidisseurs circonférentiels, notre approche est basée sur la théorie des profils ouverts en voile mince [6], [7]. Voici les principales hypothèses : (voir figure 2 et figure 3 ).

(1) Dans le cadre du contrat $n^{\circ} 1.588$ (INSA Lyon) E.D.F. - D.E.R. 


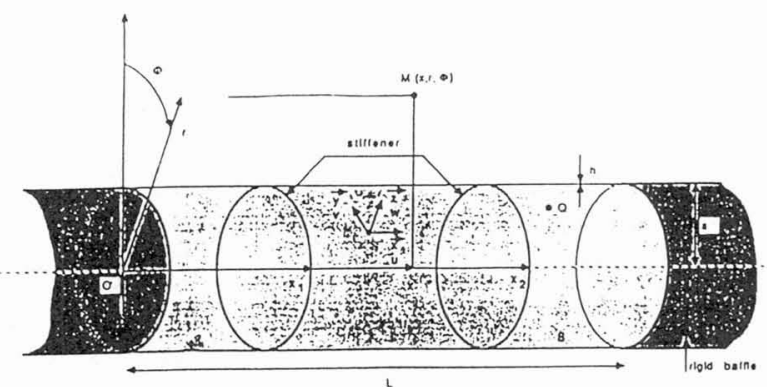

Figure 1 - Coque cylindrique raidie et système de coordonnées

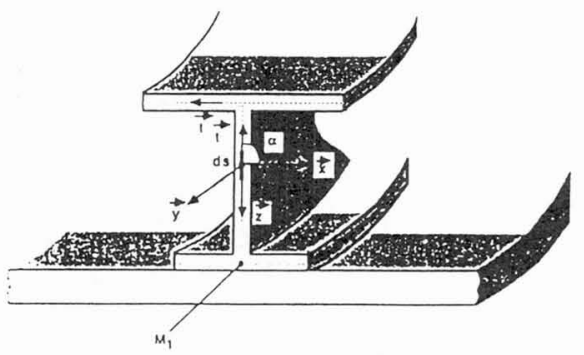

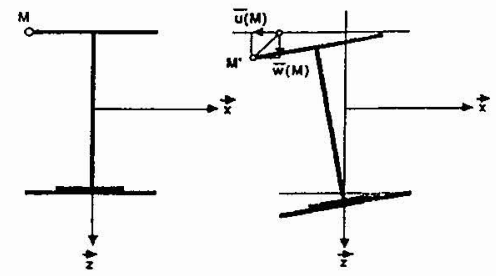

Figure 2 - Déplacement de la section droite d'un raidisseur

Figure 3 - Système de coordonnées curvilignes

1/ Dans le plan $(x, z)$ la forme de la section droite reste inchangée avant et après déformation.

2) Les contraintes de cisaillement dans le plan ( $y, t)$ sont négligeables. Ainsi le champ de déplacement d'un raidisseur de profil ouvert peut-être exprimé en "variables de coque".

$A^{\prime}$ et $M$ sont des points courants dans le plan $(x, y)$ de la section droite du raidisseur ; I est le point d'attache du raidisseur et 0 le point du feuillet moyen de la coque, il vient :

$$
\begin{aligned}
& \bar{u}(M)=u\left(x_{i}, y\right)-(z(M)-z(0)) w_{1}\left(x_{i}, y\right) \\
& \bar{w}(M)=w\left(x_{i}, y\right)+(x(M)-x(0)) w_{\prime}\left(x_{i}, y\right) \\
& \vec{v}(M)=v\left(x_{i}, y\right)+(x(I)-x(M)) u_{\prime}\left(x_{i}, y\right)+(z(0)-z(M)) w_{,}\left(x_{i}, y\right) \\
& \left.+w_{\prime} x y\left(x_{i}, y\right)\left[w_{A^{\prime}}(M, I)+z(I)-z(M)\right)\left(x\left(A^{\prime}\right)-x(I)\right)-(x(I)-x(M))\left(z\left(A^{\prime}\right)-z(O)\right)\right]
\end{aligned}
$$

avec :

$$
\omega_{A},(M, I) \text { : aire sectorielle de pôle } A^{\prime}
$$

et $u(Q)=u(x, y)-z(Q) w, x(x, y) ; v(Q)=v(x, y)-z(Q) w_{y}(x, y)$

La fonction de Hamilton est alors exprimée en "variables de coque" puisque les expressions (2) sont introduites dans (1).

Maintenant, le schéma modal de la coque non raidie étant déterminé, les déplacements de la coque raidie sont alors exprimes suivant les développements suivants :

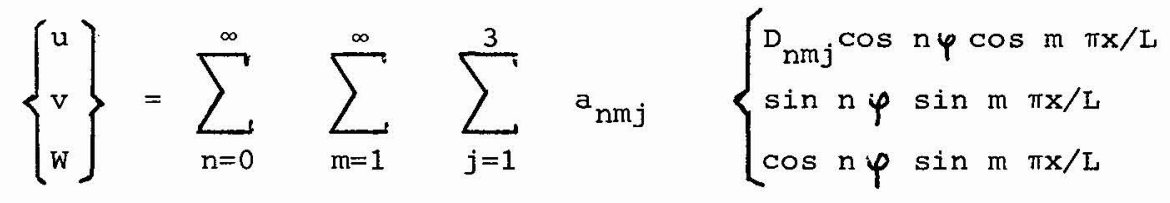


avec : I : longueur de la coque; $\mathrm{n}$ et $\mathrm{m}$, respectivement les ordres circonférentiels et longitudinaux ; $j$, type de mode (flexion, torsion, tractioncompression) ; $\mathrm{D}_{\mathrm{i}}, \mathrm{E}_{\mathrm{n}}, 1$, composantes du vecteur propre. Tous calculs effectués, la minimisąlón de la fonctionnelle de Hamilton donne les équations modales du mouvement :

$$
\begin{aligned}
& m_{p q k}\left(\omega_{p q k}^{2}-\omega^{2}\right) a_{p q k}+\sum_{i=1}^{N} \sum_{m=1}^{\infty} \sum_{j=1}^{3}\left(R_{p q m j k}^{i}-\omega^{2} M_{p q m j k}^{i}\right) a_{p m j}=0 \text { avec: } \\
& m_{p q k} \omega_{p q k}: \text { respectivement, masse généralisée et pulsation propre. } \\
& M_{p q m j k}^{i} R_{p q m j k}^{i} \begin{array}{l}
\text { respectivement, élément de la matrice de masse générali- } \\
\text { sée, et élément de la matrice de raideur généralisée } \\
\text { liés au ième raidisseur, d'abscisse longitudinale } x_{i} .
\end{array}
\end{aligned}
$$

et la forme matricielle :

(5) $\left.\left\{[m] y \omega_{s}^{2}+\left[R^{i}\right] y\right\}-\omega^{2}\{t m] y+\left[M^{i}\right] y\right\}=0$

Les pulsations propres sont les solutions de l'équation (5) et les vecteurs propres associés permettent de calculer les déformées propres.

\section{III - CALCULS-RESULTATS}

La principale difficulté numérique rencontrée lors de la mise au point du progiciel résultant est la résolution d'un système linéaire homogène c'est-àdire la résolution d'une équation du type $f(\omega)=0$. Le calcul des différents déterminants est délicat car la précision du résultat se répercute sur le calcul des vecteurs propres associés. Nous avons utilisé la méthode de Gauss avec stratégie du pivot partiel avec, lorsque cela était nécessaire du point de vue numérique, normalisation de la matrice de base.

Ici, nous proposons une comparaison des résultats obtenus par notre approche avec ceux obtenus par deux auteurs [ 1 ], [2] sur une coque raidie donnée par Hoppmann [ 1 ].

\begin{tabular}{l|ccccc}
$\mathrm{n}$ & $\mathrm{m}$ & 1 & 2 & 3 & 4 \\
\hline 2 & (a) & 1530 & 2040 & 3200 & 4440 \\
& (b) & 1565 & 2174 & 3515 & 5091 \\
& (c) & 1705 & 2301 & 3590 & 5120 \\
3 & (a) & 4080 & 4090 & 4520 & 5000 \\
& (b) & 4321 & 4312 & 4545 & 5123 \\
& (c) & 4189 & 4198 & 4449 & 5031 \\
4 & (a) & - & - & 7560 & 7800 \\
& (b) & $80 \overline{27}$ & $79 \overline{7} 2$ & 7968 & 8089 \\
& (c) & 7625 & 7579 & 7585 & 7713 \\
5 & (a) & - & - & 11400 & - \\
& (b) & $119 \overline{29}$ & $118 \overline{8} 2$ & 11840 & 11842 \\
& (c) & $1201 \overline{1}$ & 11962 & 11915 & 11910
\end{tabular}

Coque cylindrique de Hoppmann, aluminium ; rayon : 1.925 in ; épaisseur : 0.065 in ; longueur : 15.53 in ; 19 raidisseurs circonférentiels, de section rectangulaire, hauteur : $0.21 \mathrm{in}$, largeur $: 0.125$ in $\mathrm{m}$ : ordre longitudinal

$\mathrm{n}$ : ordre circonférentiel.

(a) fréquences expérimentales [1]

(b) fréquences théoriques (éléments finis) [2]

(c) notre approche.

Lors de l'exposé nous présenterons en particulier l'analyse de la densité modale d'une coque raidie, et une comparaison théorie-expérience.

\section{IV - CONCLUSION}

Nous avons présenté une méthode pour déterminer les fréquences propres et les modes propres associés d'une coque cylindrique finie et raidie circonférentiellement. Cette approche conduit à un programme de simulation simple pour déterminer le shéma modal de ce type de structure. 


\section{BIBLIOGRAPHIE}

[1] W.H. HOPPMANNII, 1958, J. Acous. Soc. Am. 30, 77. Some characteristics of the flexural vibrations of orthogonally stiffened cylindrical shells.

[2] A.M.J. AL-NAJAFI, WARBURTON G.B.,1970 J.S.V. 13(1),9-25. Free vibration of ring - Stiffened cylindrical shells.

[3] I.D. WILKEN, W. SOEDEL, 1976 J.S.V. $44(4), 563-576$. The receptance method applied to ring - Stiffened cylindrical shells : analysis of modal characteristics.

[4] D.J. MEAD, N.S. BARDELL, 1987, 115(3), 499-520. Free vibration of a thin cylindrical shell with periodic circumferential stiffeners.

[5] B. LAULAGNET, Thèse - Rayonnement acoustique des coques cylindriques finies raidies revêtues d'un matériau de masquage. 1989 INSA LYON, $190 \mathrm{p}$.

[6] VLASSOV B.z., Pièces longues en voiles minces, Paris, Eyrolles, 1962. 654 pages.

[7] BAHUAUD J. et VIALATON G., Flexion et torsion des poutres à parois minces et à section droite ouverte, INSA LYON 1980, 206 pages. 